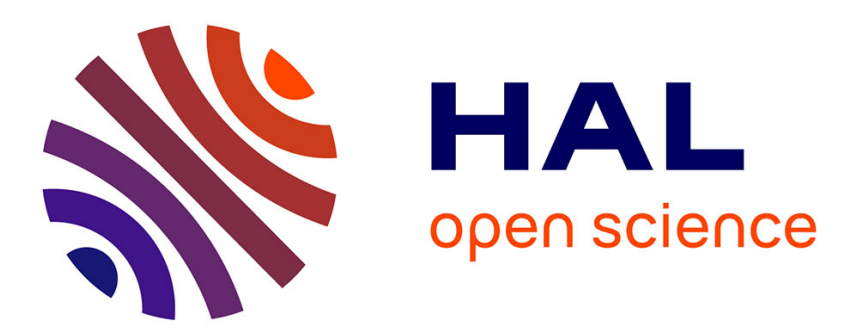

\title{
Recherche, innovation et stratégie nationale. Quand des wagons de priorités cachent la locomotive stratégique...
} Bernard David

\section{To cite this version:}

Bernard David. Recherche, innovation et stratégie nationale. Quand des wagons de priorités cachent la locomotive stratégique.... Futuribles, 2010, 360, pp.51-68. cea-00458904

\section{HAL Id: cea-00458904 https://hal-cea.archives-ouvertes.fr/cea-00458904}

Submitted on 25 Feb 2010

HAL is a multi-disciplinary open access archive for the deposit and dissemination of scientific research documents, whether they are published or not. The documents may come from teaching and research institutions in France or abroad, or from public or private research centers.
L'archive ouverte pluridisciplinaire $\mathbf{H A L}$, est destinée au dépôt et à la diffusion de documents scientifiques de niveau recherche, publiés ou non, émanant des établissements d'enseignement et de recherche français ou étrangers, des laboratoires publics ou privés. 


\title{
Recherche, innovation et stratégie nationale : Quand des wagons de priorités cachent la locomotive stratégique... Une proposition pour aller de l'avant
}

\author{
par Bernard DAVID ${ }^{1}$
}

\section{Introduction}

Le 2 décembre dernier, Valérie Pécresse ${ }^{2}$ présentait en Conseil des ministres une communication sur la stratégie nationale de recherche et d'innovation ${ }^{3}$, aboutissement d'un processus lancé par ce même Conseil des ministres le 3 septembre 2008. Cette opération faisait suite à une recommandation du Comité de modernisation des politiques publiques, "qui a décidé lors de sa réunion du 11 juin 2008 qu'un exercice de définition stratégique des priorités du Gouvernement en matière de recherche et d'innovation serait conduit tous les quatre ans, sous le pilotage du ministre de la recherche, en associant toutes les parties prenantes: membres de la communauté scientifique, milieux économiques, représentants des porteurs d'enjeux associatifs. Cette stratégie nationale sera approuvée en conseil des ministres ${ }^{4}$.

Ainsi définie comme un exercice participatif, l'opération s'est déroulée selon un schéma classique : nomination d'un Comité de pilotage prestigieux fixant un cadre aux travaux, réflexions en neuf groupes thématiques rendant chacun un rapport, consultation publique sur un site internet dédié, rédaction d'un rapport de synthèse par le ministère pilotant l'opération, enfin saisine d'un certain nombre d'instances pour avis avant l'approbation finale en Conseil des ministres. En tout six cent personnalités d'horizons variés ont été mobilisées pour cet exercice inédit en France (mais courant dans beaucoup de grands pays étrangers), dont le produit final est un document d'une trentaine de pages intitulé « Stratégie nationale de recherche et d'innovation 2009 - rapport général »"

Les différentes instances consultées pour avis ${ }^{6}$ ont unanimement salué le travail considérable effectué et souligné l'intérêt d'une telle démarche, suggérant notamment une réactualisation glissante tous les ans. Mais elles ont aussi exprimé leurs interrogations quant au caractère réellement opératoire du rapport final. Pour faire court, au-delà des observations faites sur tel ou tel aspect du rapport, elles s'interrogent sur ce que cette exercice va réellement changer en France.

Pourquoi une telle mobilisation d'éminentes personnalités ne permet-elle pas d'aboutir à la formulation d'une stratégie nationale qui apparaisse efficiente aux yeux de nombre d'acteurs et d'institutions ? C'est la question que se propose d'éclaircir le présent article. Après avoir rappelé les principales questions soulevées par ce rapport, une mise en perspective historique éclaire la confusion qui existe aujourd'hui entre trois logiques qui s'affrontent lorsqu'on parle de recherche publique. Une ouverture est proposée dans la dernière partie, en montrant comment le rapport porte en lui les ressources nécessaires pour dépasser ses limites actuelles et aller vers un dispositif plus opératoire.

\footnotetext{
${ }^{1}$ Chargé de mission prospective au Commissariat à l'énergie atomique (CEA). Les opinions exposées dans cet article n'engagent que son auteur et ne reflètent pas nécessairement l'analyse et la position du CEA.

2 Ministre de l'enseignement supérieur et de la recherche.

${ }^{3}$ Communiqué du conseil des ministres du 2 décembre 2009 : http://www.gouvernement.fr/gouvernement/la-strategienationale-de-recherche-et-d-innovation

${ }^{4}$ Communiqué du Ministère de l'enseignement supérieur et de la recherche du 3 septembre 2008 : http://media.enseignementsup-

recherche.gouv.fr/file/2008/51/9/Strategie_nationale_de_recherche_et_d_innovation_pourquoi_comment_34519.pdf

5 http://media.enseignementsup-recherche.gouv.fr/file/SNRI/69/8/Rapport_general_de_la_SNRI_-_version_finale_65698.pdf

6 Office parlementaire d'évaluation des choix scientifiques et technologiques, Académie des sciences, Académie des technologies, Conseil supérieur de la recherche et de la technologie.
} 


\section{Les principales questions soulevées par le rapport}

\section{La nature même de ce que l'on veut faire et le positionnement de l'action}

II n'est pas évident pour tout le monde que les termes recherche, innovation et stratégie nationale fassent bon ménage, et s'associent naturellement pour définir un concept précis dans un périmètre bien clair. Beaucoup de chercheurs pensent que la recherche doit avant tout être libre d'autodéterminer sa trajectoire. Quant à l'innovation, elle relève au moins autant de facteurs culturels (l'éducation), organisationnels (les institutions et leurs modes de fonctionnements) et régulatoires (règlementations, fiscalité) que d'avancées scientifiques et techniques. Enfin, dans un monde où toutes les barrières qui le compartimentaient territorialement et thématiquement s'effacent peu à peu, la notion de stratégie nationale montre ses limites et peut moins que jamais faire l'objet d'une approche 'en silo' centrée sur les seules activités de recherche et d'innovation.

C'est autour de cette question du périmètre concerné par la réflexion, donc de sa finalité, qu'apparaît la première ambigüité de la démarche entreprise: s'agit-il d'aborder les activités de recherche et d'innovation en tant que telles, ou comme moyen au service d'une stratégie globale de la nation pour assurer sa prospérité économique, sociale et intellectuelle ? Autrement dit, s'agit-il de préciser la stratégie nationale par la recherche ou pour la recherche ? Bien évidemment, la réponse est qu'il faut combiner les deux approches. Le problème est de savoir où placer le curseur, et là il semble que les avis aient divergé au sein des participants de l'opération, tout comme ils divergent aujourd'hui entre les lecteurs du rapport.

Le résultat en est un rapport final qui juxtapose l'exposé des grands défis sociétaux, des considérations relatives aux intérêts stratégiques de la nation, et une énumération des sujets de recherche qu'il faut mener en priorité.

\section{La structure du rapport et la lecture qui en est faite}

On pourrait croire, à la lumière de ce qui vient d'être observé, que chacun trouverait de quoi se satisfaire dans le rapport général. En fait, sa structure, et surtout la manière dont est affiché le sommaire (voir figure 1 ci-dessous) donnent l'impression d'être orientées vers un seul but : dresser la liste des thèmes prioritaires pour la recherche, organisés en trois grands axes : la santé, le bien-être, l'alimentation et les biotechnologies ; l'urgence environnementale et les écotechnologies ; l'information, la communication et les nanotechnologies.

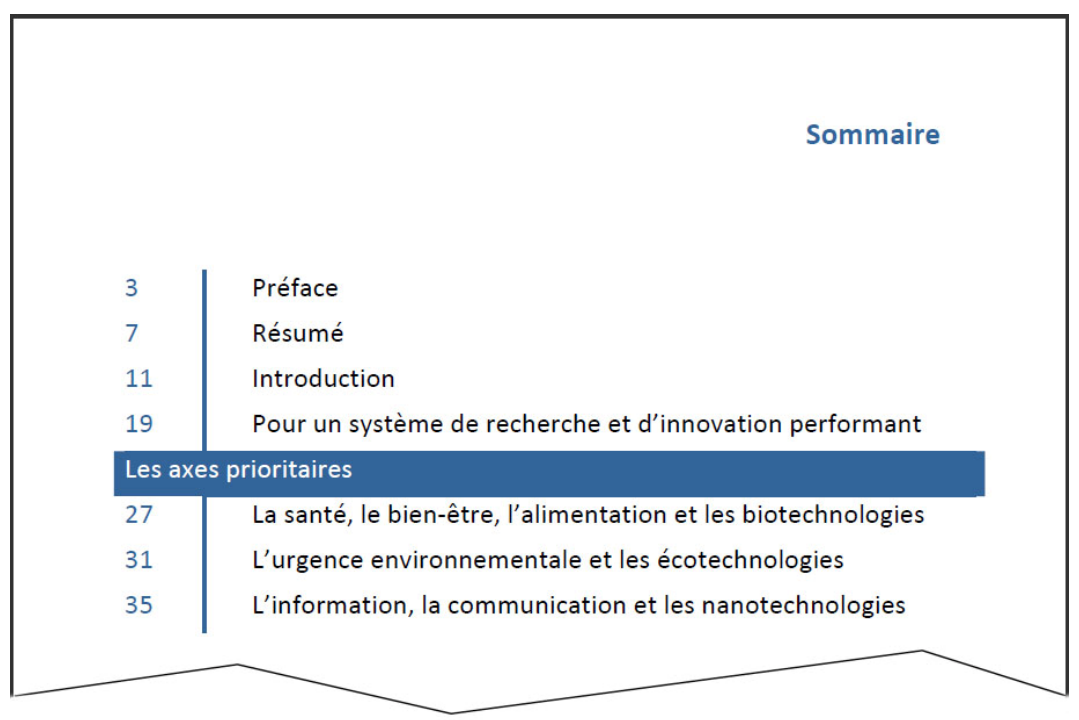

Fig. 1 : Reproduction de la page de sommaire du rapport Cette présentation suggère que le rapport est orienté vers un seul but : exposer les axes prioritaires, conduisant à une lecture tronquée de ce qu'il apporte réellement 
Dès lors, il n'est pas étonnant que les lecteurs se soient focalisés sur cette dernière partie du rapport concernant les axes prioritaires (qui représente à peine la moitié du rapport), délaissant les considérations pourtant particulièrement intéressantes de l'introduction. Cette introduction, subdivisée en plusieurs sous-parties et représentant en volume le tiers du rapport, est bien plus qu'une introduction. C'est en fait le fondement de la stratégie nationale, en particulier à travers cinq principes directeurs qui proposent une pluralité des angles de vue sur ce que représentent la recherche et l'innovation pour la nation.

Pourquoi avoir positionné ces considérations dans l'introduction ? C'est sans doute lié au processus d'élaboration de ce rapport. Les différents groupes thématiques ont essentiellement travaillé sur les contenus des recherches à mener en priorité. Fruit d'un travail collectif, ceci a sans doute été considéré comme la partie la plus noble et la plus légitime, à mettre en visibilité dans le rapport. $\mathrm{A}$ contrario, l'introduction a probablement été conçue par l'équipe projet du ministère, et de ce fait volontairement placée en position modeste, malgré son importance et sa qualité. Entre les deux est placée une courte première partie, intitulée "pour un système de recherche et d'innovation performant », dont on ne comprend pas bien comment elle s'articule dans le fil du rapport. Elle apparaît en effet à certains égards comme en retrait par rapport à ce qui a déjà été affirmé comme principes stratégiques dans l'introduction.

\section{La question des choix}

Concentrant son attention sur la partie-phare relative aux priorités thématiques, l'Académie des technologies soulève d'emblée la question centrale: «des choix ou une stratégie ? ». Bien évidemment, tout le monde est d'accord pour dire qu'une stratégie implique nécessairement des choix, mais la question soulevée est celle de la lisibilité de ces choix, de la traçabilité allant de " l'affirmation politique déterminée des orientations choisies [à] la mention des critères pris comme références pour établir ces orientations, enfin [aux] travaux ayant permis de choisir les orientations au vu des critères retenus », comme le soulignaient déjà Christian Bataille et Claude Birraux dans un autre cadre en mars $2009^{8}$.

L'avis du Conseil supérieur de la recherche et de la technologie va dans le même sens. Le CSRT «a regretté de ne pas disposer d'emblée d'une présentation argumentée justifiant de la cohérence générale de l'ensemble, notamment quant au choix des quinze thèmes : leur validité a été donnée comme un postulat, et les critères fondamentaux de leur hiérarchisation n'ont pas toujours été explicités ; les priorités sont-elles environnementales, de connaissances fondamentales, à finalité économique ou de cohésion sociale ? »?.

Cette question des choix est centrale dans le débat français sur la recherche. La pensée largement dominante en France est qu'une stratégie de recherche et d'innovation s'exprime d'abord par des choix de thématiques, donc par des réallocations de ressources publiques entre domaines d'activité. Comme chaque communauté thématique cherche à préserver ses intérêts, surtout dans la période actuelle où le système a été mis en instabilité par une succession de réformes et dispositions nouvelles, le débat est complètement bloqué.

C'est bien ce qu'observe l'Académie des technologies, qui « ne peut s'empêcher de constater qu'on y retrouve tous les sujets à la mode », conduisant à «l'impression certainement erronée que la France pourra faire tout ce que ses chercheurs ont choisi d'entreprendre $»^{10}$. A travers cette dernière remarque, l'Académie des technologies rappelle le poids qu'ont représenté les chercheurs dans les débats, malgré le souci d'ouverture à travers des personnalités du monde de l'entreprise et de la société civile. Elle précise même qu' « on ne peut s'empêcher de penser que le document stratégique a été établi sous une forte influence des chercheurs académiques qui ont privilégié des axes de long terme ».

\footnotetext{
${ }^{7}$ Avis de l'Académie des technologies sur la Stratégie Nationale de Recherche et d'Innovation, voté en séance plénière le mardi 13 octobre 2009

${ }^{8}$ Page 17 du Rapport de l'Office parlementaire d'évaluation des choix scientifiques et technologiques sur l'Evaluation de la stratégie nationale de recherche en matière d'énergie (3 mars 2009)

${ }_{9}$ Avis du Conseil supérieur de la recherche et de la technologie sur la Stratégie nationale de recherche et d'innovation, adopté en séance plénière le $1^{\mathrm{er}}$ octobre 2009 à l'unanimité (moins 3 abstentions)

${ }^{10}$ Avis de l'Académie des technologies déjà cité
} 
Le CSRT va dans le même sens, rappelant que «les conseillers [du CSRT] se sont aussi interrogés sur la composition des commissions et sur les processus de rédaction des dossiers préparatoires de la SNRI ; l'engagement des rédacteurs dans leur propre discipline [est] quelquefois trop perceptible ».

Seule l'Académie des sciences se démarque sur cette question de la polarisation excessive par le monde académique, trouvant notamment «regrettable que la recherche fondamentale ne soit pas affichée en tant qu'axe prioritaire ${ }^{11}$.

\section{La question de l'innovation}

C'est encore l'Académie des technologies qui a la formule la plus critique sur cette question de la stratégie en matière d'innovation, observant «que la rédaction associe constamment, comme un leitmotiv, les termes recherche et innovation mais que les mesures en faveur de l'innovation sont pratiquement absentes de ce document ». La question du périmètre de la réflexion et du champ d'application de la stratégie nationale, que l'on avait presque oubliée tant la question du choix des thématiques de recherche était devenue centrale, nous est ici crûment rappelée.

Si l'Académie des technologies a raison de souligner que la présentation du rapport ne fait pas la part belle aux questions d'innovation, sa position est néanmoins un peu excessive, au regard des éléments que l'on peut trouver dans la première partie du rapport, notamment dans le paragraphe «un écosystème de l'innovation plus attractif », qui rappelle la grande dynamique actuelle en matière de nouveaux instruments pour favoriser l'innovation. Mais le rapport est là victime de l'effet d'optique qu'il a lui-même choisi de créer.

II n'en reste pas moins vrai que la question de l'innovation ne peut pas être abordée dans une logique de choix thématiques, qu'elle relève pour une bonne part de politiques-cadres que les groupes de travail thématiques n'étaient pas gréés pour traiter. C'est donc tout le processus mis en place qui orientait l'exercice vers une approche «recherche», certains disant même «recherche académique », en tous cas certainement « recherche publique », puisque de recherche privée il n'est question nulle part dans le rapport.

Que le rapport soit au final plus orienté recherche qu'innovation n'est sans doute pas si gênant que cela. Ce qui l'est plus est cette polarisation des priorités vers des défis cognitifs de long terme, qui inscrit implicitement la recherche dans une logique académique, comme s'il n'en existait pas d'autres. Lever la confusion qui découle de l'usage du terme générique «recherche » apparaît dès lors nécessaire, pour mieux comprendre pourquoi les différentes parties prenantes de la recherche en France ne peuvent se satisfaire également des résultats de la démarche. Les lignes qui suivent ont pour objet d'apporter un éclairage sur cette question, en la replaçant dans une perspective historique.

\section{La notion de recherche publique mise en perspective ${ }^{12}$}

\section{La naissance du concept au $18^{\mathrm{e}}$ siècle}

La « recherche publique », en tant qu'encadrement institutionnel de pratiques scientifiques, naît en France il y a deux siècles. Bien sûr, depuis Francis Bacon (et Descartes un peu plus tard), les gouvernants avaient bien conscience de liens entre la science et le pouvoir. Mais ce n'est que dans la période pré et post-révolutionnaire française que les divers pouvoirs en place organisent systématiquement ces liens pour mettre la science au service des intérêts de l'Etat, en raison de l'impérieuse nécessité de répondre aux pressions étrangères, dans le domaine militaire en premier lieu, mais aussi dans le champ économique.

On crée en particulier les corps techniques de l'Etat et des grandes institutions scientifiques et techniques, dont des grandes écoles. L'activité scientifique se professionnalise, avec l'apparition du métier de chercheur. L'approche scientifique impulsée par les savants des corps techniques de l'Etat percole dans tout le tissu industriel qu'elle transforme profondément par l'innovation technique et

\footnotetext{
11 Commentaires de l'Académie des sciences sur le rapport "Stratégie Nationale de Recherche et d'Innovation" du ministère de l'enseignement supérieur et de la recherche (12 octobre 2009)

12 Cette partie de l'article est reprise d'un rapport de prospective interne au CEA, rédigé par l'auteur en 2007 et portant sur les devenirs possibles de l'organisation et de la gouvernance de la recherche publique en France.
} 
organisationnelle. Paradoxalement, cette situation dessert l'Académie des sciences, qui perd son monopole d'expertise scientifique et technique : placés au cœur de la société française, la science et ses usages échappent au contrôle des cercles fermés de savants.

Le modèle organisationnel français et ses dérivés qui se développeront à travers l'Europe en ce $19^{\mathrm{e}}$ siècle construiront un paysage de la recherche publique assez stable pendant une centaine d'années. Puis le début du $20^{\mathrm{e}}$ siècle est marqué en France (ce n'est pas le cas dans les pays anglo-saxons) par une prise de distance des savants vis à vis du pouvoir (à moins que ce ne soit l'inverse...) et surtout vis à vis du monde économique, notamment au nom d'une certaine éthique de la science.

Mais cette période est courte, car les deux guerres mondiales du $20^{\mathrm{e}}$ siècle, et particulièrement la seconde, fourniront l'occasion d'un nouveau resserrement des liens, en reproduisant cette situation où tout un peuple compte sur ses savants pour faire face à l'adversité. Ainsi, quelques semaines après le début de la seconde guerre mondiale, le CNRS est institué par décret du 19 octobre 1939 pour « coordonner l'activité des laboratoires en vue de tirer un rendement plus élevé de la recherche scientifique ». Le 18 octobre 1945, soit quelques mois après la fin de cette même guerre, naît le CEA en raison «de pressantes nécessités d'ordre national et international [qui] obligent à prendre les mesures nécessaires pour que la France puisse tenir sa place dans le domaine des recherches concernant l'énergie atomique ». La recherche publique au service de l'Etat est ainsi clairement réaffirmée, se renforçant jusqu'au début des années 70 avec la création de divers organismes dédiés.

\section{Le tournant du dernier quart de siècle}

Deux faits majeurs ont depuis remis en cause ce modèle relativement stable de la recherche publique au service de l'Etat. Le premier est issu du phénomène « mai 68 », c'est-à-dire de la tendance au refus de l'autorité en général et de la tutelle de l'Etat en particulier, qui conduit à réaffirmer la liberté du chercheur dans le cadre d'une nouvelle acception de la notion de recherche publique : il s'agit d'une activité produisant un bien commun de l'humanité, qui est la connaissance, et qui ne peut être confisquée au bénéfice d'intérêts particuliers, fussent-ils ceux d'un Etat. On voit ainsi réapparaître avec force le modèle de l'université médiévale, indépendante du pouvoir des Etats, et dont le pouvoir est celui de l'esprit dans le cadre d'une compétition intellectuelle internationale. Cette question de la finalité de la recherche publique reste aujourd'hui ouverte pour un grand nombre d'acteurs de la société.

Le second fait majeur remettant en cause le modèle historique est la mondialisation économique, qui fait disparaître la notion d'acteur économique national auquel le dispositif de recherche public apporterait des avantages compétitifs dans l'intérêt de la nation. Les grands acteurs économiques sont globaux, privés, et le pouvoir de la finance internationale est tel qu'une entreprise présumée « française » aujourd'hui peut être « étrangère » demain. La question de savoir comment l'Etat doit s'y prendre en matière de recherche pour défendre au mieux les intérêts vitaux de la nation est donc elle aussi largement ouverte.

\section{Le « modèle des trois logiques »}

Ainsi, la notion même de recherche publique se trouve questionnée en cette fin de $20^{\mathrm{e}}$ siècle, à la fois dans sa finalité, dans ses modalités et dans son utilité. Centrée historiquement sur une logique étatique forte, elle a toujours pris en compte des aspects économiques d'un côté, de développement des sciences de l'autre (voir figure 2 ci-dessous). Mais l'internationalisation croissante des deux logiques économique (qui devient privée) et académique, chacune ayant des règles propres de plus en plus exigeantes, combinée à un affaiblissement général de l'Etat, entraînent la recherche publique dans une spirale dispersive en hybridant la logique étatique selon des traits contradictoires qui remettent en cause sa cohérence globale.

Ce « modèle des trois logiques », développé au CEA en 2007, lui a permis de disposer de précieuses clés de lecture des dynamiques à l'œuvre au cours des trois dernières années. II permet aussi d'éclairer d'un jour nouveau les débats autour du rapport de stratégie nationale de recherche et d'innovation. 


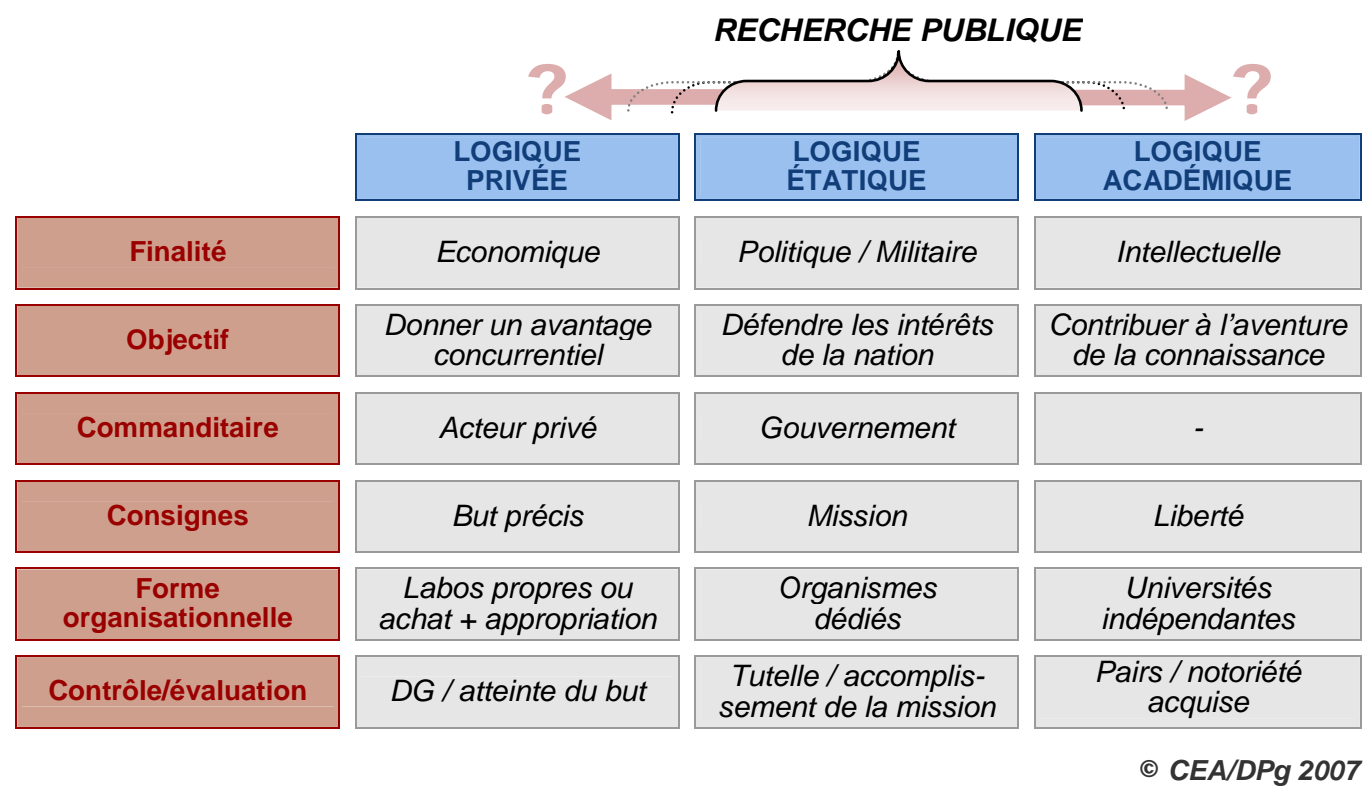

Fig. 2 : Les trois grandes logiques historiques de recherche.

La recherche publique, historiquement centrée sur la logique étatique, est aujourd'hui dans une spirale d'hybridation dispersive

\section{Des axes prioritaires au plan d'action}

\section{Comment rebondir sur ce que contient le rapport?}

Le « modèle des trois logiques », décrit précédemment, permet de comprendre pourquoi aucun des protagonistes ne peut se reconnaître totalement dans l'exercice stratégique entrepris sous l'égide du Ministère de l'enseignement supérieur et de la recherche. De manière simplifiée, on peut en effet dire que, répondant à la logique étatique, cet exercice a vu la prééminence des contributeurs académiques, tout en visant un effet d'entraînement dans la sphère privée. La confusion des logiques qui en résulte, faute d'en avoir suffisamment pris conscience, génère l'insatisfaction de chacune des parties.

Comme cela a déjà été souligné, il n'est pas dans la logique d'une collégialité académique d'effectuer des choix entre des thématiques qui toutes présentent de l'intérêt. Aller plus loin nécessite donc de mobiliser des critères relevant des deux autres logiques, pour se donner concrètement les moyens de concentrer les efforts sur ce qui apparaît le plus stratégique. II est d'autant plus important de le faire que le risque principal porté par le document actuel est de construire un cadre légitimant la poursuite de tout ce qui est déjà en cours, voire d'étendre encore la dispersion des moyens.

Ne pas « parachuter » de tels critères est évidemment fondamental pour la légitimité et crédibilité de la démarche. A ce stade de la réflexion, on se souviendra donc opportunément que le rapport comporte une partie introductive, dont l'intérêt a déjà été souligné, et qui présente des considérations susceptibles d'être transformées en de tels critères.

\section{Une caractérisation en cinq composantes}

Une des avancées du rapport est en effet d'avoir proposé cinq principes directeurs, qui précisent les exigences d'une recherche nationale selon cinq angles de vue différents, rappelés ci-après ${ }^{13}$ :

\footnotetext{
${ }^{13}$ cette reformulation concise n'engage que l'auteur du présent article ; pour le libellé détaillé, se reporter au document original
} 
- s'impliquer dans les activités de recherche fondamentale, indispensables pour comprendre le monde et pouvoir agir,

- s'ouvrir aux besoins et attentes de la société pour générer de la valeur économique et sociale par l'activité de RDI,

- prendre en compte les questions de risques pour renforcer la sécurité sous toutes ses formes,

- associer les sciences humaines et sociales aux travaux, pour créer de l'intelligibilité et du sens social,

- $\quad$ systématiser les approches pluridisciplinaires des grandes questions à traiter, afin d'innover dans leur résolution.

Ces cinq principes prennent en compte une variété des points de vue qui englobe les trois logiques de recherche précédemment évoquées. II apparaît donc pertinent d'en dériver cinq composantes de caractérisation de l'intérêt stratégique des différentes thématiques de RDI considérées comme prioritaires dans le rapport.

Néanmoins, devant le risque d'une complexité d'analyse qui interdirait au plus grand nombre d'en prendre connaissance, on a d'emblée posé comme principe que le résultat de cette caractérisation devrait être immédiatement accessible à tous par une représentation graphique.

C'est ainsi qu'a été retenue l'idée de représenter le résultat de la caractérisation de l'intérêt stratégique de chaque thème de recherche par une «image radar », sur laquelle cet intérêt stratégique serait décomposé selon cinq axes d'analyse (voir figure 3). Une fois le principe posé, la question de ce que l'on pourrait réellement représenter de pertinent et d'opératoire sur chaque axe restait évidemment entière.

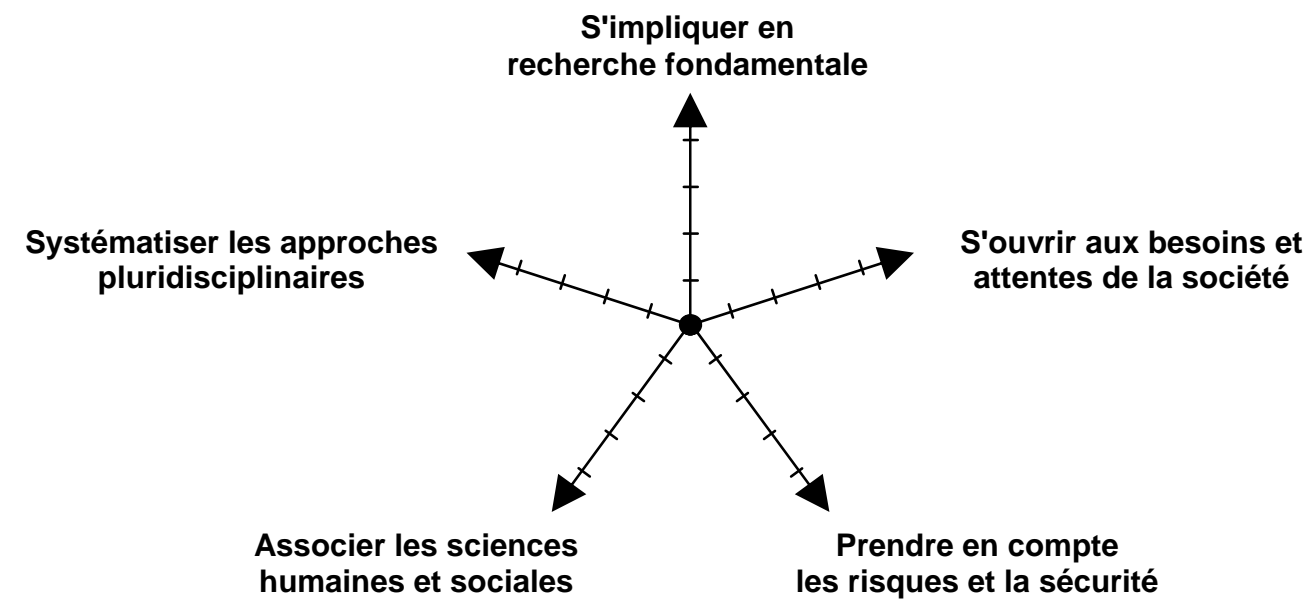

Fig. 3 : Caractérisation selon les cinq principes directeurs de la stratégie nationale

L'idée directrice est caractériser l'intérêt stratégique de chaque domaine de recherche considéré comme prioritaire relativement aux cinq 'principes

directeurs' énoncés dans la partie introductive du rapport.

\section{La nécessité d'introduire un critère de "spécificité française "}

Comme précédemment, on a recherché dans le rapport ce qui pourrait donner naissance à ce « critère d'intérêt stratégique » selon chacune des cinq dimensions d'analyse. En fait, la question est double : (1) que peut-on retenir comme critère ? (2) quelle quantification peut-on en faire ?

De manière assez paradoxale, c'est par ce qui est apparu comme un manque dans le rapport que la question du critère a trouvé sa solution. En effet, lorsque le rapport expose les raisons pour lesquelles les différentes priorités sont retenues, il entretient la confusion trop souvent faite entre les grands défis du $21^{\mathrm{e}}$ siècle, qui appellent d'importantes avancées scientifiques et techniques au plan mondial, et les 
efforts que la France peut ou doit fournir, à la fois pour contribuer aux avancées communes et pour répondre à d'impérieux besoins propres d'indépendance et de prospérité.

Il est clair que ce n'est pas la France, avec ses moyens limités, qui va relever tous les défis énumérés ici. En termes de stratégie nationale, la question doit donc plutôt être posée dans l'autre sens : dans cet effort mondial de recherche et d'innovation, sur quoi la France doit-elle se concentrer, à la fois pour être la plus utile possible à la communauté internationale, et pour préserver ses intérêts stratégiques, voire vitaux, assurant son indépendance et sa prospérité ?

Plus précisément, cette question renvoie à deux logiques et deux horizons temporels différents. La première est une logique de valorisation de la position dans laquelle elle se trouve aujourd'hui : comment, compte tenu de sa situation actuelle au plan intérieur comme international, peut-elle se saisir à court terme d'opportunités pour créer un maximum de richesse scientifique, économique, politique et sociale par son activité de RDI ? La seconde est une logique de besoin sur le long terme : sur quoi faut-il concentrer les efforts pour que la France puisse « tenir son rang » sur le long terme, en étant capable de s'affranchir de dépendances inacceptables et de s'assurer une prospérité durable ?

Se référer systématiquement et explicitement à ces spécificités du positionnement et des besoins de la France dans son environnement européen et international, est selon nous le seul moyen d'argumenter et de rendre acceptables des choix, forcément douloureux pour ceux qui les subissent, permettant de concentrer les efforts là où ils seront les plus profitables à la communauté.

C'est précisément l'objet de l'outil d'aide à la décision qui va être précisé dans les lignes qui suivent : amener ses utilisateurs, pour chaque domaine thématique retenu comme prioritaire, à établir un lien direct entre les spécificités du positionnement ou des besoins nationaux, et les modalités d'action les plus pertinentes dans ce contexte.

\section{La question de la quantification de l'intérêt stratégique}

La question de la quantification selon chacune des dimensions d'analyse est évidemment délicate. Là aussi, un certain nombre de principes ont été posés. Le premier a été de se replacer dans la logique du questionnement de la LOLF : « si je mets un euro de plus à cet endroit, qu'est-ce que je gagne ? ». L'expérience $a$ en effet montré qu'il est bien plus facile à un panel d'experts de se mettre d'accord sur les bénéfices que l'on pourrait tirer de tel ou tel effort supplémentaire par rapport à la situation d'aujourd'hui (parce qu'on peut assez facilement l'expliquer concrètement), que sur un positionnement «dans l'absolu » d'un domaine (les experts les plus proches du domaine ont systématiquement tendance à en surestimer le poids et les plus éloignés à le sous-estimer).

Le second principe a été de coter ce bénéfice par un dire d'expert(s) allant à l'essentiel, c'est-à-dire appuyé sur quelques considérations majeures, plutôt que par un travail besogneux de collecte et d'agrégation de multiples données dans lesquelles on finirait par se perdre, et qui au final ne ferait qu'ouvrir la porte à de multiples débats ou contestations à des niveaux qui n'auraient plus rien de stratégique.

Enfin, le troisième principe a été de rester dans un certain qualitatif : ce bénéfice d'un effort supplémentaire est estimé sur une échelle à quatre niveaux : faible, moyen, important, exceptionnel. Chacun des cinq axes de la représentation graphique va donc être gradué de 1 à 4 , suivant l'intensité $\mathrm{du}$ « rendement social » qu'aurait un effort supplémentaire selon cet axe.

\section{Du « profil stratégique » au plan d'action}

Une fois ces principes posés, il est vite apparu que les deux questionnements relatifs d'une part à la valorisation à court terme de la position de la France, d'autre part à la préparation de son avenir sur le long terme, devaient être abordés séparément. II a donc été décidé de juxtaposer deux «images radar », l'une relative à la valorisation à court terme de la position française et appelée «profil d'opportunité », l'autre relative aux investissements de long terme, dénommée « profil de nécessité », l'ensemble constituant le «profil stratégique » du domaine thématique examiné (les paires d'images ainsi obtenues pour les différents champs de RDI peuvent aussi être considérées comme fondant une vision stratégique en termes défensifs -profils de nécessité- et offensifs -profils d'opportunité-). 
D'aucuns trouveront cette approche beaucoup trop réductrice, utilitariste, voire égoïste face aux grands défis mondiaux qui appellent une solidarité internationale dans les efforts de recherche. Mais c'est volontairement qu'est pris ici ce contrepied, puisque l'objectif de cet outil, complémentaire de ce qui existe déjà, est de se donner les moyens d'être plus sélectifs, de choisir de façon argumentée ce qui apparaît le plus stratégique, pour en finir avec le saupoudrage subi vers des domaines dont évidemment aucun n'est totalement dénué d'intérêt, mais qui dispersent nos efforts sans générer le retour sur investissement attendu pour garantir l'avenir de nos enfants dans un monde de compétition sans pitié.

Un autre intérêt -c'est peut-être même l'intérêt majeur- de cette approche tient au fait que les cinq dimensions de caractérisation décrivent en fait des modalités d'action. Autrement dit, la mise en évidence du plan d'action le plus approprié est co-substantielle de la caractérisation du « rendement social » sur les différents axes. Ceci est d'autant plus intéressant que, même si l'analyse ne débouche sur aucune décision formelle de réorientation, les acteurs du domaine considéré auront naturellement tendance à aller vers les actions maximisant les bénéfices pour un investissement donné. En ce sens, on peut dire que cet outil ouvre une nouvelle perspective d'amélioration du rendement du système de recherche et d'innovation français, en contournant les blocages relatifs aux réallocations de ressources entre domaines.

\section{Mise en œuvre de cet outil de caractérisation}

L'approche retenue conduit à faire appel à un niveau d'expertise alliant une bonne connaissance « de l'intérieur » du domaine et une certaine hauteur de vue, de manière à pouvoir mobiliser et exprimer rapidement les quelques arguments majeurs qui fondent une appréciation d'intime conviction entre les quatre niveaux pour chaque composante de l'analyse. Ceci correspond assez bien au profil des participants des groupes de travail thématiques, ayant œuvré en amont du rapport général.

On a donc cherché en premier lieu dans les documents produits par les groupes si les éléments de la caractérisation souhaitée pour les différents domaines retenus comme prioritaires pouvaient en être extraits. Deux observations ont pu être faites à cette occasion. La première est que l'explicitation des analyses fondant les choix de priorités est très inégale selon les rapports, ce qui montre bien que les reproches de traçabilité insuffisante depuis les analyses jusqu'aux choix sont assez fondés. Un seul rapport de groupe déroule formellement une analyse de type «SWOT ${ }^{14}$. La seconde observation est que ces analyses ne produisent pas une argumentation du type de ce qui est attendu pour renseigner les « images radar».

L'idée a alors germé que cet outil de caractérisation allait peut-être forcer ses utilisateurs à développer une nouvelle gamme d'arguments, qui enrichiraient le débat et ouvriraient sur de nouvelles capacités d'action. C'est ce caractère innovant potentiel qu'a exploré une rapide expérimentation, menée en interrogeant deux experts sur deux domaines thématiques d'actualité. L'encadré de la page suivante donne plus d'éléments sur cette expérimentation, dont les résultats ont semblé très prometteurs.

Bien évidemment, un tel outil a aussi des limites d'utilisation (par exemple il doit s'intéresser à un périmètre assez homogène), il peut conduire à des mésinterprétations des diagrammes (la surface ne représente aucunement le poids d'un domaine), etc. Aller au-delà de ce qui est proposé ici, pour en faire un outil réellement opératoire, nécessite une appropriation par ceux qui pourraient être amenés à l'utiliser, appropriation qui passe sans doute par des adaptations, modifications ou raffinements, conservant l'esprit et les avantages de cet instrument sans en conserver nécessairement la forme. L'appel aux bonnes idées est en tout cas lancé.

\section{Conclusion}

On ne soulignera jamais assez l'intérêt et l'importance d'avoir mis en place un processus de concertation d'une telle ampleur pour construire une stratégie nationale de recherche et d'innovation, que l'on aura tout loisir d'améliorer au fil des ans, surtout si le principe de la réactualisation glissante est retenu. Bien sûr, la question de l'articulation aux autres modalités de gouvernance stratégique de la nation est à examiner : comment retrouve-t-on les priorités énoncées dans la loi de finance ?

\footnotetext{
${ }^{14}$ Strengths, Weaknesses, Opportunities and Threats, soit en français : forces, faiblesses, opportunités et menaces.
} 
Comment cette stratégie de recherche et d'innovation est-elle prise en compte dans les états généraux de l'industrie? Etc.

S'arrêter au stade du rapport général issu de ce processus présente néanmoins le risque de perpétuer une dispersion des efforts sur de trop nombreuses thématiques de recherche. II faut donc agir dès maintenant, pour transformer cette stratégie en un plan d'action permettant de concentrer les efforts sur ce qui va créer le plus de richesse économique, sociale et cognitive, à la fois sur le court et le long terme.

Le détour par deux siècles d'histoire du concept de «recherche publique » a montré pourquoi le rapport n'avait pas pu aller plus loin dans la question des choix en mêlant, sans assez l'expliciter, plusieurs logiques de recherche aux caractéristiques assez différentes. La proposition d'un outil d'aide à la décision, permettant de caractériser l'intérêt stratégique de chacun des domaines thématiques retenus comme prioritaires, en découle. Cet outil est d'autant plus prometteur qu'il débouche directement sur les éléments d'un plan d'action concret, en désignant les modalités d'action au meilleur « rendement social ».

Le facteur essentiel de succès d'une telle approche est la capacité de concentrer le regard sur les singularités nationales génératrices d'opportunités et de nécessités, ce qui n'est pas toujours aisé. Ceci suppose en particulier une très bonne connaissance à la fois du terrain en France et de la situation internationale, que ce soit dans une perspective européenne ou mondiale.

Cette nécessité d'être à la fois à un niveau stratégique et très précis dans l'argumentation a conduit à formuler une argumentation qui ne se retrouve ni dans le rapport général, ni dans les rapports plus détaillés des groupes de travail thématiques. Ceci conduit à penser qu'il s'agit d'une approche porteuse d'innovation: en mobilisant d'autres argumentaires, on ouvre la voie à de nouvelles capacités de gouvernance.

Enfin, la posture introduite par cet outil permet de dépasser le blocage actuel lié au dogme selon lequel mettre en œuvre une stratégie nationale passe d'abord par une réallocation des ressources entre domaines thématiques, dans un contexte d'incertitude face à l'avenir, où chacun s'arc-boute sur ses acquis pour ne rien changer.

II suggère une première étape où l'on montre que l'on peut déjà faire bien mieux avec ce que l'on $a$, par des inflexions volontaristes à l'intérieur de chacun des domaines, directement déduites de l'analyse faite par les acteurs eux-mêmes, donc propice à les mettre en mouvement et à préparer de futures étapes.

Il ouvre aussi la voie à d'autres approches macroscopiques du pilotage de la stratégie nationale, moins « intrusives » dans les domaines d'activité, fondées sur des actions différenciées par familles de profils stratégiques. 
Encadré (sur double page)

\section{Première expérimentation de l'outil d'aide à la décision}

L'objectif de l'expérimentation était de tester la capacité de l'outil proposé à amener l'utilisateur à formuler une argumentation de nature nouvelle, très synthétique tout en étant très spécifique de la situation française, pour conduire à un "visuel " immédiatement suggestif de l'orientation souhaitable des efforts nationaux de RDI.

On a donc interrogé deux experts sur deux domaines thématiques d'actualité fin 2009 : les batteries pour l'industrie automobile et l'imagerie médicale. Compte tenu de l'objectif essentiellement méthodologique à ce stade, on n'a pas cherché à valider leur analyse en la croisant avec celle d'autres experts du domaine. II est bien évident que, dans un usage normal de l'outil, on interrogerait un panel d'experts pour avoir un avis consolidé. Le lecteur ne s'attachera donc pas au fond des arguments, qui peuvent refléter un point de vue discutable, mais à leur nature et à la manière de les utiliser.

Après avoir expliqué à l'expert ce que l'on attendait de lui, on a procédé sous forme d'un entretien semi-directif, d'une durée d'environ une heure. La première question posée portait sur les enjeux particuliers que l'expert voyait pour la France, motivant un engagement national sur cette thématique. Il est frappant de voir que chacun des deux experts a d'abord mobilisé des arguments non spécifiques de la situation française, expliquant pourquoi ce thème était important pour le devenir du monde (arguments du type de ceux que l'on retrouve dans le rapport). Ce n'est que dans un second temps, en le poussant dans ses retranchements, que l'expert répond précisément à la question de la spécificité nationale, et il le fait alors très bien.

Une fois cette spécificité exprimée, l'expert n'a aucun mal à répondre à la seconde question, en désignant celle(s) des cinq dimensions d'action sur laquelle (ou lesquelles) il faut s'investir prioritairement. On peut alors passer au troisième point, qui est de proposer une quantification des bénéfices que l'on peut attendre d'efforts supplémentaires selon chacune des modalités d'action. Dans la pratique, cette troisième étape a présenté quelques difficultés. De compréhension d'abord, puisqu'il est demandé un rapport bénéfice/coût d'efforts supplémentaires par rapport à ce qui est déjà fait aujourd'hui («si je mets un euro de plus sur cette modalité d'action par rapport à ce qu'on fait aujourd'hui, qu'est-ce que je pense gagner ? ») ; d'acceptabilité du résultat ensuite, parce que si on a déjà beaucoup fait par le passé, il n'est peut-être pas utile d'en faire plus, ce qui veut dire qu'on va coter très bas cet axe, avec le risque qu'un lecteur distrait en déduise qu'il s'agit là d'une thématique peu importante.

Néanmoins, une fois l'exercice terminé, les experts se sont déclarés satisfaits de la démarche intellectuelle et de son exigence, les poussant à sortir des argumentaires habituels pour explorer une nouvelle manière d'analyser la situation. Le résultat des deux entretiens, sous forme très synthétisée, apparaît sur les 'profils stratégiques' représentés ci-dessous.

\section{Synthèse de l'entretien avec l'expert $\mathrm{N}^{\circ} 1$ : \\ Le profil stratégique du domaine thématique « batteries pour automobiles »}

L'industrie automobile est un secteur d'activité essentiel pour la France. Y survivre suppose de se positionner sur les voitures électriques. Le modèle économique de la voiture électrique met la batterie au cœur. Un enjeu majeur est d'imposer une norme technologique avancée avant la norme « de fait » des productions de masse asiatiques. Trois priorités d'action en découlent : avant tout renforcer les liens entre recherche et industrie pour mieux maîtriser les performances des batteries en conditions d'utilisation réelles; utiliser toutes les ressources amont permettant de «faire la différence »; développer des technologies sûres et le prouver. La pluridisciplinarité découle de ces priorités. L'implication des SHS deviendra importante pour faire le lien entre ce que permettront les batteries et les modalités d'usage qui en découleront ('business model'). S'agissant d'un domaine qui n'a pas atteint sa maturité, l'investissement stratégique prime sur la capacité de valoriser les atouts actuels (comme la capacité de mettre à disposition des moyens lourds de la recherche amont pour caractériser les batteries), ce qui explique que le profil de nécessité prévaut sur le profil d'opportunité. 


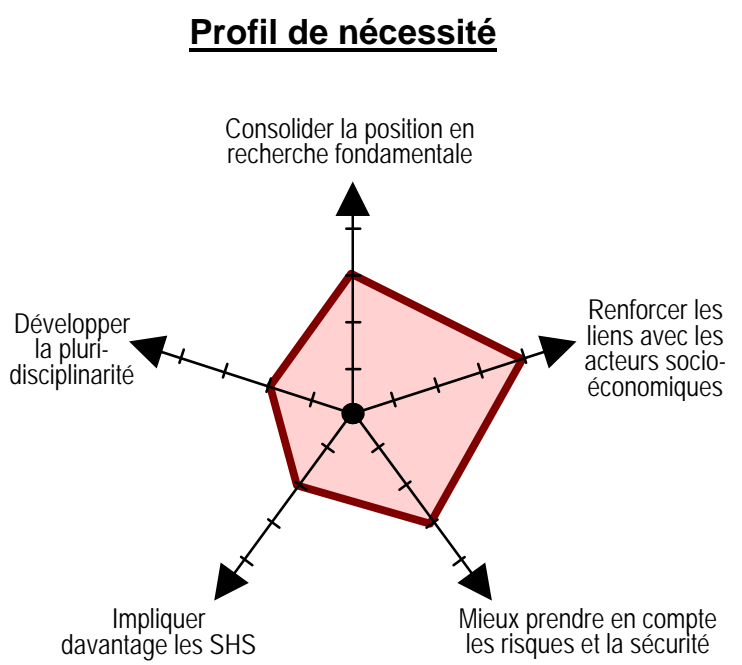

\section{Profil d'opportunité}

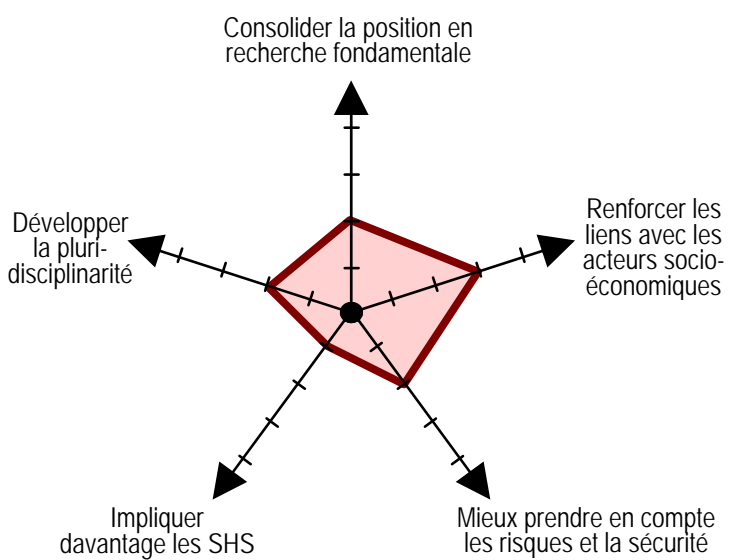

Synthèse de l'entretien avec l'expert $\mathrm{N}^{\circ} 2$ :

Le profil stratégique du domaine thématique « imagerie médicale »

L'imagerie médicale correspond à des marchés importants pour le futur, mais sans champions nationaux. Nos plateformes de recherche avancée ont une certaine attractivité pour les « majors » du domaine, par leur capacité de générer des innovations dans une course permanente aux performances et fonctionnalités nouvelles : profiter de cette opportunité nécessite avant tout de renforcer nos liens avec l'ensemble des parties prenantes de l'imagerie médicale, de développer encore la pluridisciplinarité avec un fort accrochage à la recherche amont, afin d'être très réactifs. II ne faut pas pour autant négliger la préparation de l'avenir, dans la mesure où les progrès de l'imagerie ouvrent sur la médecine individualisée, le «théranostic » et la pharmacie de demain (aidons nos champions nationaux à s'y positionner). Sans atteindre le niveau du profil d'opportunité, le profil de nécessité comporte donc deux axes forts du côté de la recherche amont et pour la prise en compte des questions de risques et de sécurité. Ces axes forts n'étant pas les mêmes que pour le profil d'opportunité, la question de l'arbitrage entre le court et le long terme se trouve posée.

\section{Profil de nécessité}

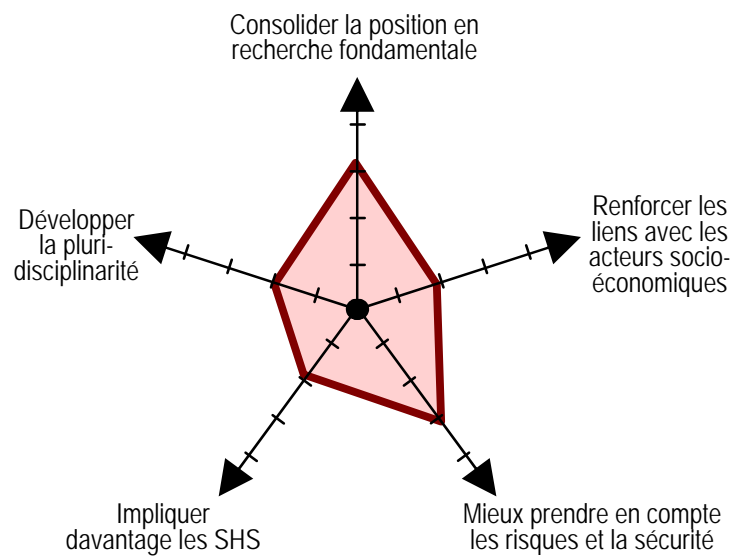

\section{Profil d'opportunité}

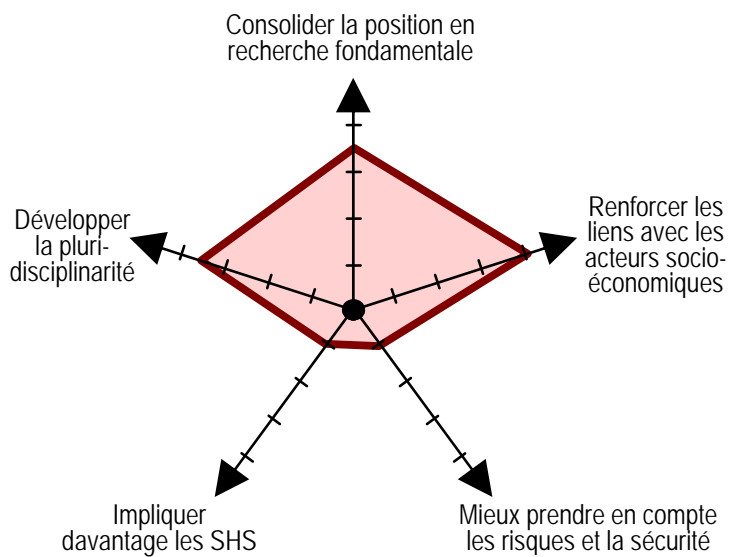

